\title{
ACREDITACIÓN DE COMPETENCIAS DE LOS ORIENTADORES PROFESIONALES EN CONTEXTOS NO ESCOLARES: EL PROYECTO EUROPEO EAS (European Accreditation Scheme)
}

\author{
ACCREDITATION OF CAREER GUIDANCE PRACTITIONES WORKING \\ OUTSIDE THE SCHOOL CONTEXT: EUROPEAN PROJECT EAS \\ (European Accreditation Scheme)
}

\author{
Elvira Repetto Talavera*, Ma José Mudarra Sánchez, Nuria Manzano Soto, \\ Maite Uribarri González y Consuelo Vélaz de Medrano Ureta \\ Universidad Nacional de Educación a Distancia
}

\begin{abstract}
RESUMEN
El proyecto europeo EAS financiado por el Programa Leonardo da Vinci diseña y valida un sistema europeo de acreditación en competencias para profesionales de la orientación en contextos no escolares. En él se definen los siguientes objetivos: 1) Revisar los sistemas de acreditación de los orientadores europeos, 2) Desarrollar un listado de competencias que conforman un sistema de acreditación en 17 países europeos, 3) Realizar un estudio piloto con 350 orientadores y 4) Elaborar el Manual Explicativo del sistema de acreditación propuesto. Para ello, se sigue una metodología cualitativa y cuantitativa y se utilizaran diversas fuentes de recogida de datos. Las conclusiones señalan la existencia de una necesidad real y pertinencia de los sistemas de acreditación a nivel europeo.
\end{abstract}

Palabras clave: Acreditación en competencias, Orientadores profesionales, Cualificaciones del orientador y Perfil profesional del orientador.

\section{ABSTRACT}

The European project EAS has been financed by the Leonardo da Vinci program with the aim of developing and validating an European frame of basic professional standards - a common accreditation system - for those working as career practitioners outside the school context. Four phases along two

\footnotetext{
* Elvira Repetto Talavera (erepetto@edu.uned.es). Ma José Mudarra Sánchez. Nuria Manzano Soto. Maite Uribarri González. Consuelo Vélaz de Medrano Ureta. Departamento MIDE II (Orientación Educativa, Diagnóstico e Intervención Psicopedagógica) de la Facultad de Educación, UNED.
} 
years were necessary, with the following objectives: 1) Revision of the existing systems of accreditation for guidance practitioners, 2) Development of a list and a system of accreditation of competencies for guidance practitioners, 3) Pilot study through 350 practitioners in 17 European countries, and 4) Development of the Users Manual for the accreditation system obtained. A mixed methodology (qualitative and quantitative), different sources of data gathering (online questionnaire, personal surveys and review of documentation) and several samples were used for the pilot study. For the descriptive analysis, an intended sampled was chosen.

The results obtained are a common system of competencies accreditation for guidance practitioners and a user's manual. Conclusions point the existence of a real need for such accreditation systems and the contribution of this proposal to fill the gap for this type of tools.

Key Words: Competencies accreditation, Career Guidance Practitioner, Counselor Professional Qualification, Counsellor Professional Profile.

\section{Introducción}

Dentro del Programa Leonardo da Vinci (ref. IT/06/C/F/TH-81406), el proyecto EAS dirigido en España por E. Repetto, se desarrolla entre Octubre de 2006 y Septiembre de 2008 con la participación de los siguientes socios: Institute of Careers Guidance (ICG) y Canterbury Christ Church University (CCCU) de Inglaterra, Consorzio per la Ricerca e l'Educazione Permanente (COREP) y FORCOOP Agenzia Formativa, de Italia, KADIS de Eslovenia, y la Universidad Nacional de Educación a Distancia (UNED) y la Asociación Española de Orientación y Psicopedagogía (AEOP), desde España.

El objetivo principal es desarrollar y validar un marco europeo común de estándares profesionales para quiénes ejerciesen actividades de orientación profesional en contextos no escolares.

La pertinencia de este proyecto radica en la necesidad y dificultad de una definición de las cualificaciones y la formación del orientador en el contexto europeo. La delimitación del perfil profesional del orientador constituye un reto significativo que responde a la necesidad institucional de definir sus roles y competencias profesionales. Si bien es prolija la literatura disponible sobre modelos de intervención orientadora (Rodríguez Espinar, 1993; Rodríguez Moreno, 1983, 1992; Repetto, 1998 y 2002; Sanz Oro, 2001; Solé, 1998; Malik, 2002; Vélaz de Medrano 1998 y 2002, entre otros) sorprende la escasez de investigaciones aplicadas que contrasten las propuestas teóricas y evalúen las dificultades de la función orientadora en la práctica. De hecho algunos estudios constatan (Lázaro y Mudarra, 2000) cómo en la práctica profesional no existe aún suficiente diferenciación de funciones y competencias orientadoras, detectándose cierto distanciamiento entre los perfiles genéricos de competencias y sus aplicaciones en el ejercicio profesional.

Así mismo, Watts \& Van Esbroeck (1998) tras analizar los sistemas de orientación y asesoramiento dentro de las instituciones europeas de educación superior cuestionan la identidad profesional del orientador al identificar roles y servicios en los que se realizaban distintos tipos de tareas que exigían diferentes tipos de experiencia. El proyecto EAS se ubica en el Marco europeo de cualificaciones para el aprendizaje permanente (CE, 2008), - EQF-MEC - un marco común de referencia para facilitar la movilidad profesional y el acceso al aprendizaje permanente. Ahora bien, para lograr un esquema común de estándares 
profesionales es preciso que todos los socios de este proyecto compartan una misma terminología, más allá de diferencias en los tipos y duración de los programas de formación de orientadores profesionales en cada país, e incluso entro de ellos, entre diversos sectores profesionales (en instituciones educativas y formativas, servicios públicos de empleo y empresas tanto en el sector público como privado). El esfuerzo por delimitar conceptos y consensuar terminologías se inicia con un desafío semántico: el concepto de competencia.

Es frecuente la confusión conceptual derivada de documentos oficiales en los que se intercambian los términos de competencies - aptitudes específicas de entrada - y competences - realizaciones globales, resultados- (Sultana, 2009), así como la distinción entre competencias fundamentales - core competences - y especializadas - specialized competences-. Tras analizar numerosas definiciones (Le Boterf, 2001; OCDE, 2002; Sobrado, 2004; De Miguel et al, 2005; Lázaro y Mudarra, 2005; Rodríguez Moreno, 2006; Repetto et al, 2007; Echeverría, 2008; CE, 2008; CEDEFOP, 2009), se observa la preocupación por los elementos que deberían conformar el concepto de competencia (incluyendo también aspectos internos más difíciles de valorar, De Miguel, 2005, resaltando la dimensión intencional, observable y situacional, LeBotef, 2001) y cómo deberían integrarse de forma dinámica y sistémica — movilización de recursos - para dar respuesta eficaz a demandas complejas - que exigen saber, querer y poder actuar, Echeverría, 2008 - surgidas en un contexto determinado (capacidad adaptativa de las competencias de la que dependerá el "desempeño efectivo de la actividad profesional", Rodríguez Moreno, 2006; Sobrado, 2008, la obtención de un resultado óptimo, Repetto et al, 2007). Tal especificidad contextual influirá en la secuencia concreta de competencias que se consideren indicadores de la profesionalidad del orientador dependientes a su vez del enfoque paradigmático desde el que se orienta (Lázaro y Mudarra, 2005).

Desde la dirección del proyecto EAS, se simplifica el concepto de competencia definiéndola como la principal actividad identificable y estable que el orientador profesional debía lograr. En la tabla 1 se exponen algunas de las definiciones terminológicas adoptadas.

El sistema de acreditación debe procurar la comprensión de quienes ofrecen orientación profesional en diversos contextos sin olvidar la forma que en cada país adoptan los servicios de orientación profesional, variable según tradiciones, valores culturales y corrientes socio-económicas. EAS reconoce muchas actividades de orientación, tareas fundamentales - main tasks —, que forman parte de un perfil profesiográfico más amplio. Los cinco principios claves de EAS que guian el desarrollo de EAS y dirigen su aplicación se recogen en la tabla 2.

Los contextos de aplicación de EAS, fundamentalmente "no escolares" son: (a) servicios de orientación para poblaciones específicas, (b) servicios educativos -incluyendo centros públicos y privados, universidades y sistemas a distancia-, (c) agencias implicadas en formación profesional dirigidas a empleados y demandantes de empleo, adultos y grupos específicos, (d) servicios de empleo públicos y privados, (e) departamentos de recursos humanos dentro de organizaciones y empresas y, (f) servicios de información, asesoramiento y orientación vía internet.

En consecuencia, los destinatarios de EAS, son personas con diversos perfiles profesionales (Career Guidance Practitioners), que pueden ejercer tareas fundamentales de orientación profesional: orientadores vocacionales, orientadores educativos, asesores personales, especialistas en información profesional, profesionales de la rehabilitación profesional, es- 
pecialistas en inserción y desarrollo profesional, tutores y coaches en el ámbito empresarial, asesores comunitarios, agentes de inclusión social etc. (Repetto et al, 2007). Los beneficios potenciales tras la aplicación de EAS se recogen en la tabla 3.

TABLA 1. Definiciones terminológicas adoptadas en el Proyecto EAS.

\begin{tabular}{|l|l|}
\hline \multicolumn{1}{|c|}{ Término } & \multicolumn{1}{c|}{ Definición } \\
\hline $\begin{array}{l}\text { Accredit } \\
\text { (Acreditar) }\end{array}$ & $\begin{array}{l}\text { Certificar y dar constancia de algo de una de forma oficial y con autoridad } \\
\text { reconocida. }\end{array}$ \\
\hline $\begin{array}{l}\text { Career Guidance } \\
\text { (Orientación } \\
\text { Profesional) }\end{array}$ & $\begin{array}{l}\text { Rango de procesos diseñados para capacitar a individuos en la toma de } \\
\text { decisiones informada y en sus transiciones -relativas al aprendizaje o al } \\
\text { trabajo-. }\end{array}$ \\
\hline $\begin{array}{l}\text { Competence } \\
\text { (Competencia) }\end{array}$ & Capacidad para realizar algo bien. \\
\hline $\begin{array}{l}\text { Competenes/ies } \\
\text { (Competencias) }\end{array}$ & $\begin{array}{l}\text { Conjunto de conocimientos y características personales relacionados con el } \\
\text { buen desempeño de un trabajo específico. }\end{array}$ \\
\hline $\begin{array}{l}\text { Main task } \\
\text { (tarea } \\
\text { fundamental) }\end{array}$ & $\begin{array}{l}\text { Debe ser (a) identificable, (b) tener entidad en sí misma, (c) exigir en su } \\
\text { realización destrezas y conocimientos significativos y específicos y, (d) } \\
\text { relevante. Ej.: rellenar un formulario a partir de una entrevista no se consideraría } \\
\text { tarea fundamental, sin embargo sí lo sería proporcionar información sobre } \\
\text { orientación profesional, como una actividad independiente realizada cara a } \\
\text { cara en una entrevista. }\end{array}$ \\
\hline $\begin{array}{l}\text { Task } \\
\text { (Tarea) }\end{array}$ & $\begin{array}{l}\text { Unidad de trabajo específica o conjunto de actividades necesarias para obtener } \\
\text { cierto rendimiento. Ej.: escribir un resumen de una entrevista. }\end{array}$ \\
\hline $\begin{array}{l}\text { Qualification } \\
\text { (Cualificación) }\end{array}$ & $\begin{array}{l}\text { Un individuo se considera cualificado para un puesto de trabajo en función de } \\
\text { sus credenciales o experiencia - o ambas- }\end{array}$ \\
\hline $\begin{array}{l}\text { Skill } \\
\text { (destreza) }\end{array}$ & $\begin{array}{l}\text { Habilidad para hacer algo bien. Ej.: La realización correcta del resumen de una } \\
\text { entrevista implica poseer aptitudes lingüísticas. }\end{array}$ \\
\hline
\end{tabular}

TABLA 2. Principios del EAS (adapt. Reid, 2008).

\begin{tabular}{|l|}
\hline \multicolumn{1}{|c|}{ Principio } \\
\hline $\begin{array}{l}\text { 1. La relevancia de realizaciones basadas en tareas más que en jerarquías de aprendizaje o expe- } \\
\text { riencia. }\end{array}$ \\
\hline 2. La importancia de reconocer el aprendizaje no formal. \\
\hline 3. La necesidad de plantear prácticas de evaluación pertinentes acordes con la tarea y su contexto. \\
\hline 4. La necesidad de flexibilidad, unida a la garantía de calidad de un proceso riguroso. \\
\hline $\begin{array}{l}\text { 5. La exigencia de coherencia con los planteamientos de desarrollo de orientación profesional en } \\
\text { Europa y en un contexto más amplio, con el marco europeo de cualificaciones y formación. }\end{array}$ \\
\hline
\end{tabular}


Elvira Repetto Talavera et al. Acreditación de competencias de los orientadores profesionales ...

TABLA 3. Beneficios potenciales de EAS (adapt. Reid, 2008).

\begin{tabular}{|c|c|}
\hline Para individuos & Para organizaciones \\
\hline $\begin{array}{l}\text { Desarrollar un sentimiento de autoconfianza y } \\
\text { eficacia. }\end{array}$ & Reconocer la competencia del staff. \\
\hline $\begin{array}{l}\text { Medir destrezas y competencias conforme a es- } \\
\text { tándares europeos. }\end{array}$ & $\begin{array}{l}\text { Desarrollar descripciones de ocupaciones y es- } \\
\text { pecificaciones de carácterísticas personales. }\end{array}$ \\
\hline $\begin{array}{l}\text { Recibir feedback constructivo sobre la propia } \\
\text { práctica profesional — tareas fundamentales-. }\end{array}$ & Planificar necesidades del staff. \\
\hline Mejorar el modelo de práctica profesional. & Mantener políticas de contratación. \\
\hline $\begin{array}{l}\text { Enriquecer el curriculum vitae y el perfil profe- } \\
\text { sional. }\end{array}$ & Identificar y solventar necesidades formativas. \\
\hline $\begin{array}{l}\text { Obtener la acreditación en ciertas tareas funda- } \\
\text { mentales. }\end{array}$ & Desarrollar apoyos y tutorías para el staff. \\
\hline $\begin{array}{l}\text { Ampliar posibilidades de reconocimiento profe- } \\
\text { sional en otros sectores y países. }\end{array}$ & $\begin{array}{l}\text { Asegurar la calidad de servicios y atención diri- } \\
\text { gidos a clientes. }\end{array}$ \\
\hline $\begin{array}{l}\text { Demostrar un compromiso continuo con el pro- } \\
\text { pio desarrollo profesional. }\end{array}$ & $\begin{array}{l}\text { Emplear criterios externos y enfoques transpa- } \\
\text { rentes con el staff. }\end{array}$ \\
\hline \multirow[t]{4}{*}{$\begin{array}{l}\text { Vincularse a una red europea de prácticos de la } \\
\text { orientación profesional. }\end{array}$} & $\begin{array}{l}\text { Mantener una fuerza de trabajo capacitada, mo- } \\
\text { tivada, confiada en sí misma y flexible. }\end{array}$ \\
\hline & $\begin{array}{l}\text { Ajustar objetivos individuales y grupales a las } \\
\text { metas de la organización. }\end{array}$ \\
\hline & $\begin{array}{l}\text { Demostrar la competencia de la organización a } \\
\text { entidades externas o servicios de inspección. }\end{array}$ \\
\hline & $\begin{array}{l}\text { Vincularse a entidades profesionales y asocia- } \\
\text { ciones europeas. }\end{array}$ \\
\hline
\end{tabular}

\section{Método}

El proyecto EAS (2006-2008), se ha desarrollado a través de las siguientes fases (véase tabla 4):

Durante la Fase 1, tanto los equipos participantes como los expertos elaboraron un informe en el que se recogen los procedimientos de evaluación y reconocimiento de la competencia para los orientadores 18 países de la OCDE. Se solicitó un breve informe a otros expertos pertenecientes a diferentes países de la OCDE, incluyendo cuestiones referentes al perfil del orientador, sistemas de acreditación para la obtención de credenciales profesionales, entidades expendedoras de dicha acreditación y requisitos mínimos de acceso y su evaluación.

Además de los documentos previamente señalados, se revisaron las aportaciones de los principales esquemas de acreditación en un marco internacional, tales como el EVGP (Educational and Vocational Guidance Practitioner), el GCDF "Global Career Development Facilita- 
tor" y el NCCC "National Certified Career Counselor”, el programa del CACREP "Council for Accreditation of Counseling and Related Educational Programs" el CMP "Career Management Professionales" el "Canadian National Steering Committee for Career Development Guidelines and Standards" y el sistema australiano (CICA). En el ámbito europeo, se analizó con especial detalle las aportaciones de las National Vocational Qualifications (NVQs).

TABLA 4. Fases y diseño metodológico del Proyecto EAS.

\begin{tabular}{|c|c|c|c|}
\hline Fases & $\begin{array}{c}\text { Entidad y país } \\
\text { responsable }\end{array}$ & Objetivo específico & Método/técnica \\
\hline 1 & $\begin{array}{l}\text { CCCU } \\
\text { (Reino Unido) }\end{array}$ & $\begin{array}{l}\text { Revisar la literatura existente para ana- } \\
\text { lizar los sistemas de acreditación para } \\
\text { orientadores existentes en los países } \\
\text { de la OCDE, con especial atención a } \\
\text { los requisitos de acceso y métodos de } \\
\text { evaluación. }\end{array}$ & Análisis de contenido. \\
\hline 2 & $\begin{array}{l}\text { UNED } \\
\text { (España) }\end{array}$ & $\begin{array}{l}\text { Desarrollar una taxonomía original de } \\
\text { competencias para los orientadores } \\
\text { profesionales y un sistema para su } \\
\text { acreditación. }\end{array}$ & Análisis descriptivo. \\
\hline 3 & $\begin{array}{l}\text { KADIS } \\
\text { (Slovenia) }\end{array}$ & $\begin{array}{l}\text { Coordinar un estudio piloto del esque- } \\
\text { ma de acreditación con un mínimo de } \\
350 \text { orientadores y } 17 \text { organizaciones } \\
\text { en } 17 \text { países europeos. }\end{array}$ & $\begin{array}{l}\text { Simulación del procedi- } \\
\text { miento de acreditación } \\
\text { real. } \\
\text { Análisis comparativo de } \\
\text { distintos informantes. } \\
\text { Análisis descriptivo. }\end{array}$ \\
\hline 4 & $\begin{array}{l}\text { CCCU } \\
\text { (Reino Unido) } \\
\text { Todos }\end{array}$ & $\begin{array}{l}\text { Elaborar un Manual explicativo del sis- } \\
\text { tema de acreditación obtenido. } \\
\text { Difundir el esquema de acreditación re- } \\
\text { sultante. }\end{array}$ & \\
\hline
\end{tabular}

Durante la Fase 2, se diseña un mosaico de competencias y un sistema de acreditación para orientadores.

Tras la presentación de diversas propuestas y la integración de las opiniones se elabora un documento definitivo que contiene las cinco tareas principales que pueden evaluarse de manera independiente así como un grupo de elementos comunes que debían ser evaluados para cada tarea principal en la que el candidato solicitara la acreditación. Además se estableció un proceso de evaluación basado en la revisión de documentación del candidato (títulos, certificados, declaración del empleador, contratos, y otros) y una entrevista personal (ver epígrafe Resultados).

La Fase 3 del proyecto desarrolla un estudio piloto con el esquema de acreditación que incluye un mínimo de veinte participantes por cada país. Los candidatos que participan, no pretenden obtener la acreditación sino probar el sistema de acreditación. Los países socios decidieron utilizar la vía telemática como medio preferente de: a) enlace a los cuestionarios de solicitud y de información académica y profesional, y b) entrega de documentación de los sujetos participantes (títulos o certificados que pudieran ser considerados parte de la evidencia). 
Los evaluadores estudian sus formularios de acreditación y documentación clasificándolos en dos grupos (candidatos con una experiencia inferior vr superior a 1.000 horas en el campo profesional de la orientación) así como concertar entrevistas presenciales, telefónicas o vía Internet. La evidencia de la competencia del candidato en todos los elementos contenidos dentro de la(s) tarea(s) en la(s) que deseaba ser evaluado (incluyendo los elementos comunes) se codifica como suficiente o insuficiente. Caso de no hallar evidencias concluyentes y suficientes de su competencia (obtenidas en los dos últimos años), son contactados de nuevo para solicitar evidencias adicionales. En los casos en que las evidencias aportadas por el candidato no cumplieron estas premisas, el evaluador identificó y recomendó al candidato posibles itinerarios de formación adicional o una mejora de la experiencia profesional.

Por último el feedback sobre el proceso se obtiene también por vía telemática. Se diseñan cuestionarios para recoger información cuantitativa y cualitativa de los evaluadores que valorara la claridad de los documentos y el sistema de acreditación en sí mismo. Asimismo, también se elaboran cuestionarios específicos para entidades representativas del sector, tales como Asociaciones, Sindicatos, instituciones públicas y otros, así como para potenciales entidades concesionarias de la Acreditación EAS en un futuro.

\section{Resultados}

Tras la elaboración de una sintesis conceptual sobre los enfoques de competencias y sus Sistemas de acreditación en los países de la OCDE (su exposición excedería el propósito de este artículo) que permitiese identificar cuestiones clave en las que debía posicionarse EAS (¿Qué definición y/o modelo de competencias era deseable?, ¿Qué competencias o tareas de los orientaciones profesionales eran más relevantes?, ¿Qué métodos de evaluación eran los más adecuados?, ¿Qué tipo de acreditación era más coherente?), se obtuvo como resultado el desarrollo del esquema de acreditación EAS (mosaico de competencias y proceso de acreditación) cuya versión final adopta la siguiente estructura.

1. Principales actividades y áreas de orientación profesional importantes para el usuario y suficientemente flexibles para permitir diversidad de actividades orientadoras (tabla 5).

2. Cinco tareas principales de orientación profesional ${ }^{1}$ sobre las que se realiza el estudio piloto (como consecuencia del mismo se reducen a tres, véase tabla 7). Asimismo, se identifican los elementos comunes a todas las tareas de orientación profesional (tabla 6) entendidos como elementos esenciales que deben guiarlas - evaluables separadamente-.

3. Elementos especificos - evaluables - que integran las tareas principales (tabla 7). La acreditación en una tarea conlleva la evaluación tanto de sus elementos comunes como específicos.

4. Fases del Proceso de evaluación y acreditación.

\footnotetext{
1. Estas tareas debían seguir los criterios de: a) identificable, b) tener entidad en sí misma, c) exigir en su realización destrezas y conocimientos significativos y específicos, y d) relevante.
} 
TABLA 5. Actividades y áreas de orientación profesional consideradas para un "esquema de tareas principales" (Evangelista, 2007b).

1. Identificar y proporcionar información sobre orientación profesional tanto cara a cara como a distancia.

2. Recoger, organizar, difundir información sobre orientación profesional para usarla individualmente.

3. Diseñar materiales de información para uso del servicio de orientación.

4. Identificar las necesidades del cliente y derivarlo cuando sea necesario.

5. Realizar actividades de consejo y orientación individualmente con cada usuario.

6. Realizar actividades de consejo y orientación a distancia con los usuarios.

7. Establecer redes eficazmente.

8. Dar seguimiento y apoyo a personas que están en proceso de búsqueda de empleo y/o itinerarios educativos, de forma individual.

9. Dar seguimiento y apoyo a personas que están en proceso de búsqueda de empleo y/o itinerarios educativos en pequeños grupos.

10. Mediación

11. Diseñar actividades de educación para la carrera.

12. Facilitar cursos de técnicas de búsqueda de empleo.

13. Facilitar otro tipo de actividades de educación para la carrera en pequeños grupos.

14. Diseñar otros programas o servicios.

15. Coordinar programas o servicios.

TABLA 6. Elementos comunes evaluables en cada tarea.

B.1. Respetar los principios éticos.

B.2. Establecer y mantener un clima con los usuarios (incluidos la comunicación eficaz) para maximizar la eficacia de la relación de orientación.

B.3. Demostrar compromiso con la mejora continua del conocimiento, de las propias destrezas y del servicio.

B.4. Establecer relaciones de trabajo apropiadas con todas las personas implicadas en el proceso de orientación, ya sean internas o externas a la organización.

B.5. Utilizar las TIC para los propósitos de orientación profesional.

B.6. Conocer cómo y dónde encontrar información sobre orientación profesional.

Fase 0. Solicitud del procedimiento y admisión: Se estudia la evidencia que permitirá al evaluador decidir si acepta o no la solicitud del candidato, evaluado únicamente en las competencias demostrables. El candidato solicita la acreditación de al menos una de las tareas que ha ejercido su trabajo durante un mínimo de 3 años, referidas a las áreas de Diagnóstico y evaluación, Gestión de la información; Intervención para el desarrollo de la carrera y seguimiento; Formación para la carrera; Interacción, coordinación y contacto con otros profesionales e instituciones. Para ello debe aportar el impreso de admisión y un portfolio que incluye (véase tabla 8).

Y seguir las siguientes fases (véase tabla 9). 
TABLA 7. Tareas principales y elementos específicos a evaluar en EAS.

\begin{tabular}{|c|c|}
\hline $\begin{array}{l}\text { Tarea principal } \\
\text { (Main task) }\end{array}$ & Elementos específicos \\
\hline $\begin{array}{l}\text { 1. Organizar información } \\
\text { sobre orientación pro- } \\
\text { fesional como una ac- } \\
\text { tividad separada. }\end{array}$ & $\begin{array}{l}\text { C.1.1. Recoger, organizar, producir y difundir información sobre orien- } \\
\text { tación profesional. } \\
\text { C.1.2. Organizar información sobre orientación profesional, cara a } \\
\text { cara y/o a distancia, y/o en grupos. }\end{array}$ \\
\hline $\begin{array}{l}\text { 2. Realizar entrevistas de } \\
\text { orientación profesio- } \\
\text { nal. }\end{array}$ & $\begin{array}{l}\text { C.2.1. Utilizar técnicas de entrevista para realizar entrevistas. } \\
\text { C.2.2. Evaluar las necesidades de los usuarios. } \\
\text { C.2.3. Seleccionar y utilizar herramientas de evaluación de la carrera } \\
\text { cuando es necesario. } \\
\text { C.2.4. Atender a los usuarios para determinar un plan de acción. } \\
\text { C.2.5. Asistir a los usuarios para llevar a cabo el plan de acción pre- } \\
\text { visto. } \\
\text { C.2.6. Gestionar los casos de orientación asignados. }\end{array}$ \\
\hline $\begin{array}{l}\text { 3. Llevar a cabo activida- } \\
\text { des de orientación } \\
\text { profesional con peque- } \\
\text { ños grupos. }\end{array}$ & $\begin{array}{l}\text { C.3.1. Utilizar técnicas de grupo para facilitar pequeños grupos de } \\
\text { usuarios (30). } \\
\text { C.3.2. Atender a usuarios para llevar a cabo transiciones de aprendi- } \\
\text { zaje, de formación o de trabajo (incluyendo búsqueda de traba- } \\
\text { jo). } \\
\text { C.3.3. Atender usuarios para desarrollar, implementar y revisar su } \\
\text { plan de acción. }\end{array}$ \\
\hline
\end{tabular}

TABLA 8. Elementos a incluir el portfolio.

Currículum europeo (información personal, campo de trabajo, experiencia laboral, formación y competencias y habilidades personales y profesionales).

Documentos oficiales que acrediten la formación.

Documentos oficiales que acrediten la práctica.

Cuestionario de autoevaluación de competencias.

Documento de adhesión al código ético.

En la evaluación se propone la triangulación metodológica: además de la revisión de la documentación aportada, el evaluador debe incluir, al menos, un método de evaluación directa, ya sea entrevista personal, discusión profesional u observación además puede optar por otros como, el feedback de terceras personas (empleadores, colegas y clientes), la evaluación de productos, la simulación, etc. Respecto a la validez del esquema de acreditación EAS, por razones de espacio, en este artículo sólo se describe la realización del estudio piloto (ver epígrafe Método) y su producto final, (esquema EAS), abierto a un continuo proceso de validación.

Finalmente, otro resultado es el Manual explicativo de EAS o guía para la acreditación de orientadores profesionales, con los aspectos clave para realizar una acreditación de competencias del orientador profesional (Reid \& Ford, 2008). 
TABLA 9. Fases del proceso de evaluación y acreditación.

\begin{tabular}{|l|l|}
\hline $\begin{array}{l}\text { I. } \\
\begin{array}{l}\text { Evaluación de la so- } \\
\text { licitud de acredita- } \\
\text { ción }\end{array}\end{array}$ & $\begin{array}{l}\text { Se analiza la solicitud de acreditación. El asesor comunica al candidato } \\
\text { la admisión o rechazo de su solicitud y las competencias susceptibles } \\
\text { de acreditación }\end{array}$ \\
\hline $\begin{array}{l}\text { II. } \\
\text { Planificación del } \\
\text { procedimiento de } \\
\text { evaluación }\end{array}$ & $\begin{array}{l}\text { Se especifica la estrategia de evaluación. El evaluador planifica el pro- } \\
\text { ceso y acuerda con el candidato la agenda de evaluación, explicándole } \\
\text { el proceso. }\end{array}$ \\
\hline $\begin{array}{l}\text { III. Recopilación y regis- } \\
\text { tro de evidencia }\end{array}$ & $\begin{array}{l}\text { Se reúne la evidencia necesaria acreditativa de la formación recibida y } \\
\text { la práctica en las competencias a evaluar. Se archivará toda la informa- } \\
\text { ción relativa al proceso: identidad del candidato y el evaluador, planifi- } \\
\text { cación, métodos utilizados y conclusiones parciales. }\end{array}$ \\
\hline $\begin{array}{l}\text { IV. Decisión de acredi- } \\
\text { tación }\end{array}$ & $\begin{array}{l}\text { Se juzga si la evidencia recopilada es suficiente para acreditar la tarea a } \\
\text { evaluar. }\end{array}$ \\
\hline $\begin{array}{l}\text { V. Informar al candida- } \\
\text { to }\end{array}$ & $\begin{array}{l}\text { Se le informa sobre la decisión final y el resultado del proceso, a saber: } \\
\text { acreditación positiva (certificación), necesidad de aportar evidencia adi- } \\
\text { cional, acreditación negativa (identificando las deficiencias y aconsejan- } \\
\text { do rutas formativas necesarias para obtener la acreditación. }\end{array}$ \\
\hline $\begin{array}{l}\text { VI. Verificar la calidad } \\
\text { de la decisión }\end{array}$ & $\begin{array}{l}\text { Se evalúa la calidad de la decisión adoptada y la interpretación correcta } \\
\text { de los estándares. Para ello podrán designarse evaluadores externos } \\
\text { del proceso. }\end{array}$ \\
\hline
\end{tabular}

\section{Conclusiones}

Como indica el informe de la OECD (2004), en la mayor parte de los países, la orientación está poco profesionalizada. También en nuestro país, los orientadores profesionales carecen de un perfil delimitado, y por tanto, se hace necesario regularizar su formación y acreditación, en aras a la mejora de la calidad de los servicios de orientación y la protección de sus clientes o usuarios. A diferencia de los orientadores que ejercen su tarea en instituciones educativas, los que trabajan en otros ámbitos, ya sea empleo o inserción socio-comunitaria carecen de un itinerario formativo claro y requisitos de acceso establecidos. Al tratarse además de profesionales con una trayectoria profesional muy variada, parece indicado apostar por un sistema de acreditación de la experiencia adquirida por la vía no formal, de manera que se evalúen y certifiquen aquellas competencias que posee el orientador en el momento de solicitar la acreditación, y que han sido adquiridas mediante la práctica. En nuestro país esto ya se viene haciendo para las titulaciones de Formación Profesional mediante la definición del Catálogo Nacional de Ocupaciones.

La definición del perfil profesional de los orientadores es actualmente uno de los objetivos prioritarios de distintos organismos a nivel nacional e internacional, ya sean sindicatos, instituciones educativas o asociaciones profesionales, aunque coinciden en definir este perfil sobre un modelo basado en competencias, discrepan en cuanto a la formación inicial y continua que sería deseable o exigible. Otros problemas surgen, a la hora de facilitar un procedimiento riguroso, a la vez que viable, para evaluar y acreditar dichas competencias.

Precisamente, el objetivo del modelo de evaluación y acreditación de competencias de los orientadores profesionales propuesto, es desarrollar y probar unos estándares mínimos 
de acreditación de competencias para orientadores profesionales. Se ha diseñado teniendo en cuenta el contexto de la orientación profesional, considerando la transferibilidad y portabilidad de la certificación obtenida por el profesional, de modo que le acredite para ejercer tareas de orientación similares en cualquier país de la Unión Europea. Se espera que esta propuesta de acreditación de orientadores profesionales contribuya a llenar un vacío en la oferta de sistemas de acreditación a nivel europeo, cubriendo una necesidad real para acreditar a estos profesionales que trabajan en los contextos no escolares.

Entre los aspectos más positivos destaca el esfuerzo realizado por identificar una estructura común de acreditación de competencias a escala europea que permita a los orientadores, organizaciones e instituciones formativas describir un perfil de tareas clave de orientación profesional sobre los que trabajar. No obstante, el equipo español considera, como limitación, que esta aportación requiere mayor consenso entre los países participantes para salvar dificultades en su aplicación derivadas de las diferencias conceptuales y las prácticas de orientación vigentes en cada país. Así mismo, es fundamental un estudio piloto para probar su viabilidad real, avalar su validez a través de instituciones oficiales, a nivel nacional y europeo garantizando con ello la aplicabilidad y utilidad del modelo propuesto.

Como prospectiva, para mejorar la propuesta EAS, el equipo español está trabajando sobre el siguiente modelo matricial (figura 1).

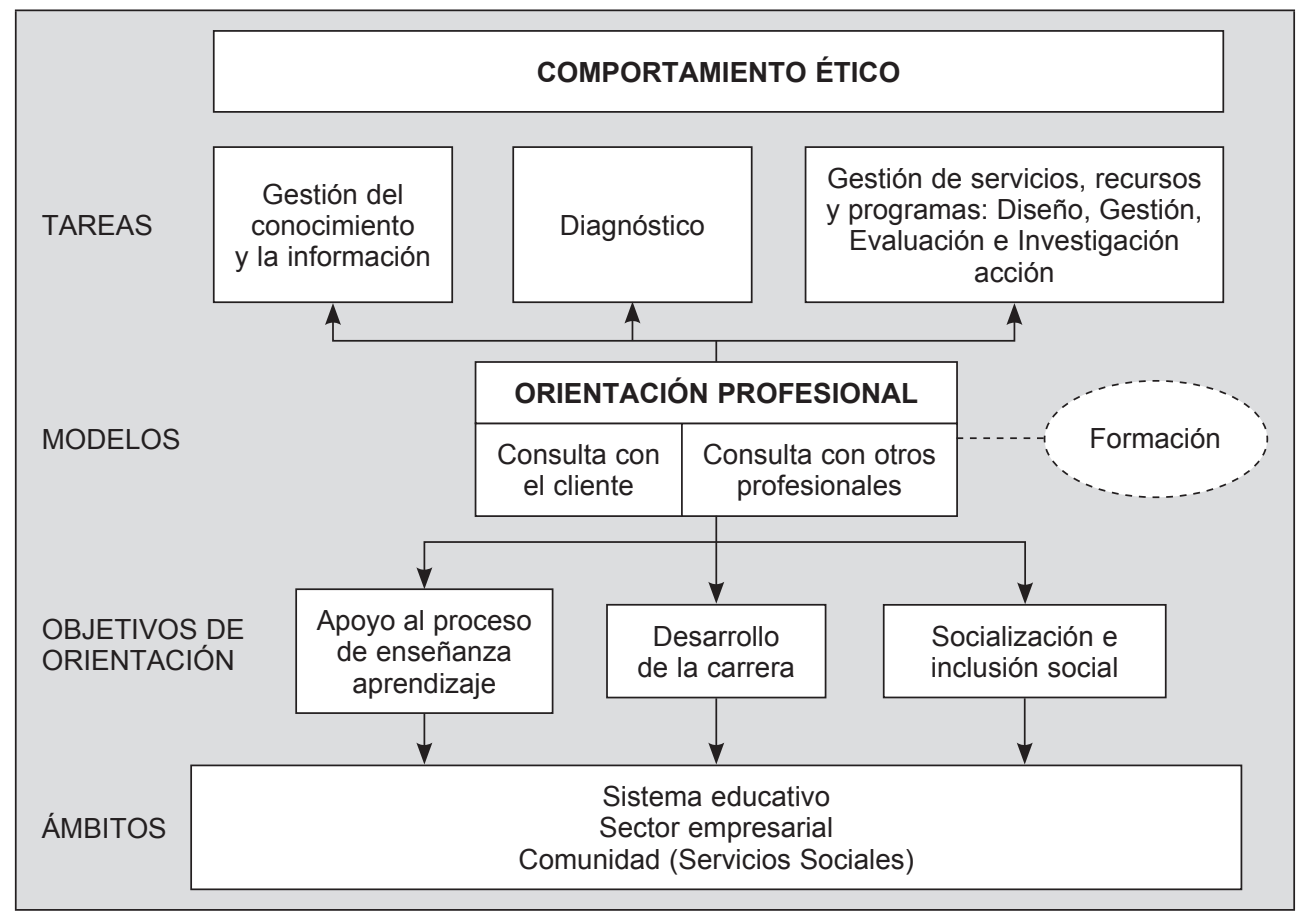

FIGURA 1.

Modelo matricial de orientación profesional. 


\section{Referencias Bibliográficas}

CCE-IAEVG (2007). Application for Educational and Vocational Guidance Paractitioner: EVGP. Greensboro, NC: Center for Credentialing and Education-IAEVG.

CE (2008). El Marco Europeo de Cualificaciones para el Aprendizaje Permanente (EQF-MEC) http://ec.europa.eu/dgs/education_culture/publ/pdf/eqf/broch_es.pdf. Accesible en junio de 2009.

CEDEFOP (2009). Professionalising career guidance: Practitioner competences and qualification routes in Europe. Luxembourg: European Centre for the Development of Vocational Training.

CICA (2006). Profesional estándars for Australians Career Development Practitioners. National Standards and Accreditation of Career Practitioners Project. Australia: Department of Education, Science and Training \& Career Industry Council of Australia.

Clarke, L. \& Winch, Ch.(2006). A European skills framework?-but what are skills? Anglo-Saxon versus German concepts. Journal of Education and Work Vol. 19, No. 3, July 2006, pp. 255-269.

De Miguel Díaz, M. (Dir) ; Alfaro Rocher, I. J.; Apodaca Urquijo, P.; Arias Blanco, J. M.; García Jiménez, E.; Lobato Fraile, C. y Pérez Boullosa, A. (2005). Modalidades de enseñanza centradas en el desarrollo de competencias: Orientaciones para promover el cambio metodológico del Espacio Europeo de Educación Superior. Oviedo: Ediciones Universidad de Oviedo-MEC.

Echeverría, B.; Isús, S.; Martínez Clares, P. y Sarasola, L. (2008). Orientación profesional. Barcelona: UOC.

ENTO (2006). Assessment Strategy for the Qualifications based on the National Occupational Standards for Advice and Guidance. Leicester, UK: The National Trainning Organization for Employment ENTO.

ETUC, UNICE/UEAPME and CEEP (2002). Framework of Actions for the Lifelong Development of Competencies and Qualifications, Brussels: ETUC, UNICE/UEAPME and CEEP, 28 February.

Hiebert, b. (2000). Competencies for providing quality career services: a look at Canadian standars for career development Revista Española de Orientación y Psicopedagogía, vol 11, 19, 5-19.

Lázaro, A. y Mudarra, M. J. (2005). Las competencias del orientador en la movilidad profesional. Revista Qurriculum, 18, 175-191.

Lázaro, A. y Mudarra, M. J. (2000). Análisis de los estilos de Orientación en los Equipos Psicopedagógicos. Contextos Educativos, vol 3, 253-289.

Le Boterf, G. (2001). Ingeniería de las competencias. Barcelona: Gedisa.

Lévy-Levoyer, C. (1997). Gestión de competencias. Cómo analizarlas, cómo evaluarlas, cómo desarrollarlas. Barcelona: Gestión 2000.

National Steering Committee for Career Development Guidelines and Standards (2004). Canadian Standards and Guidelines for Career Development Practitioners. Ottawa: Author. http://www. career-dev-guidelines.org.

OCCOPPQ (2004). Guidance Counsellors's General Competency Profile. Montreal: OCCOPPQ.

OECD (2004). Career guidance: a Handbook for Policy Makers. París: OECD.

Reid, H. (2007). Study on the Accreditation Schemes of OECD Countries: wp1 Final Report for website $\mathrm{http}: / / \mathrm{www} . c 0 r e p . i \mathrm{t} / \mathrm{eas} / \mathrm{home}$.htm.

Reid, H. \& Ford, A. (2008). Guide on the Accreditation of Careers Guidance Practitioners: putting the EAS into practice. European Commision: EAS-Leonardo da Vinci Program.

Repetto, E. y Vélaz, C. (1998). Orientación de la carrera, recursos humanos y mercado laboral. Actas y congresos. UNED, Madrid.

Repetto, E. (2002). Modelos de Orientación e Intervención Psicopedagógica. Madrid, UNED. 
Repetto, E., Mudarra, M. J., Uribarri, M., Ferrer, P., Vélaz de Medrano, C. y Manzano, N. (2007). Accreditation Scheme. Informe preparado para el Proyecto Leonardo da Vinci "European Accreditation Scheme for Career Guidance Practitioners". http://www.corep.it/eas/home.htm.

Repetto, E.; Ferrer-Sama, P.; Manzano Soto, N. y Hiebert, B. (2008). "International competencies for educational and vocational guidance practitioners". International Journal Educational and Vocational Guidance, 8, 135-195.

Rodríguez Espinar, S.; Álvarez, M.; Echeverría, B. Marín. A. (1993). Teoría y práctica de la orientación educativa. Barcelona: PPU.

Rodríguez Moreno, M. L. (2008). El perfil de competencias del informador profesional. Revista Española de Orientación y Psicopedagogía, vol 19, nº 2, 235-239.

Rodríguez Moreno, M. L. (2006). De la Evaluación a la Formación de Competencias Genéricas: Aproximación a un modelo. Revista Brasileira de Orientaçao Profissional, 7 (2), 33-48.

Rodríguez Moreno, M. L. (1992). El mundo del trabajo y las funciones del orientador. Barcelona: Barcanova.

Rodríguez Moreno, Ma Luisa; Gil Bussalleu, Teresa-Natalia. (1983). Modelos de intervención educativa y vocacional. Barcelona: PPU.

Sanz Oro, R. (2001). Orientación psicopedagógica y calidad educativa. Madrid: Pirámide.

Sobrado, L. (2004). Evaluación y Orientación de competencias y cualificaciones profesionales. Barcelona: Estel.

Sobrado, L.; Couce, A. y Rial, R. (2003). Exploración de las competencias de los orientadores profesionales en contextos sociolaborales. Revista Española de Orientación y Psicopedagogía, vol 14, $\mathrm{n}^{\mathrm{o}} 2,41-62$.

Solé, I. (1998). Orientación educativa e intervención psicopedagógica. ICE/Horsori, Barcelona.

Sultana, R. G. (2009). Competence and Competence Frameworks in Career Guidance: complex and contested concept. International Journal for Educational and Vocational Guidance, 9, pp. 15-30.

TWG (2003). Credit Transfer in VET, First Report of the Technical Working Group, October 2003. http://cedefop.communityzero.com/credit-transfer.

Vélaz de Medrano, C. (1998). Orientación e Intervención Psicopedagógica: concepto, modelos, programas y evaluación. Málaga, Aljibe.

Vélaz de Medrano, C. y Repetto, E. (1999). "Lo que piensan los orientadores de la Comunidad de Madrid sobre su ejercicio y desarrollo profesionales". En Actas del Congreso Internacional de Psicología y Educación sobre Orientación e Intervención Psicopedagógica. Asociación de Psicología, Educación y Psicopedagogía (APEP). Santiago de Compostela, pp. 358-359.

Vélaz de Medrano, C., Repetto, E., Blanco, A., Guillamón, JR, Negro, A. y Torrego, JC. (2001). "Evaluación de las necesidades de desarrollo profesional de los orientadores de Educación Secundaria”. Revista de Investigación Educativa (RIE), vol. 19, nº 1, pp. 199-220.

Winterton, J. Delamare-Le Deist, F. \& Stringfellow, E. (2005). Typology of knowledge, skills and competences: clarification of the concept and prototype. CEDEFOP-Thessaloniki: Centre for European Research on Employment and Human Resources, Group ESC Touluse.

Fecha de recepción: 10-10-2009

Fecha de revisión: 19-10-2009

Fecha de aceptación: 21-10-2009 